\title{
Classificação do uso e cobertura da terra utilizando imagens SAR/Sentinel 1 no Distrito Federal, Brasil
}

\author{
Land Use/Land Cover (LULC) classification based on SAR/Sentinel 1 image in \\ Federal District, Brazil
}

\author{
Felipe Lima Ramos Barbosa ${ }^{1}$ \\ Renato Fontes Guimarães ${ }^{2}$ \\ Osmar Abílio de Carvalho Júnior ${ }^{3}$ \\ Roberto Arnaldo Trancoso Gomes ${ }^{4}$
}

\begin{abstract}
Palavras-chave:
SAR

Sentinel 1

Coerência interferométrica

Ambiente urbano

Objetos urbanos
\end{abstract}

\section{Keywords:}

SAR

Sentinel 1

Interferometric coherence

Urban environment

Urban objects

\begin{abstract}
Resumo
Os desafios sociais e ambientais estão diretamente relacionados à concentração populacional existente nos ambientes urbanos, que contribuem em mais de $75 \%$ do Produto Interno Bruto (PIB) mundial. Assim, é essencial a detecção da dinâmica do uso e cobertura da terra visando à elaboração e efetivação de políticas públicas. Os sistemas SAR, especialmente as técnicas de interferometria, vêm apresentando ótimos resultados frente a esse desafio, uma vez que não possuem influência direta da atmosfera. Limitados estudos foram realizados utilizando-se a coerência interferométrica proveniente do satélite Sentinel-1 em ambiente urbano. Nesse sentido o objetivo desse estudo foi classificar o uso e cobertura da terra de parte do Distrito Federal tendo por base diferentes dimensionalidades considerando as medidas de intensidade e de coerência interferométrica relativas ao ano de 2018. Os resultados mensurados a partir das métricas Kappa e F1 indicam que a inserção de uma série temporal de coerências interferométricas melhora o desempenho da classificação, de 0,50 a 0,75 (Карpa) e de 0,54 a 0,79 (F1), fato que ficou evidente na melhora de desempenho das classes temáticas relacionadas à cobertura vegetal. Ademais, também constata-se que a identificação dos objetos urbanos é melhor representada pela utilização apenas das intensidades (VV e VH) no processo classificatório.
\end{abstract}

\footnotetext{
${ }^{1}$ Universidade de Brasília, Departamento de Geografia, Brasília, DF, Brasil. felipelrb@gmail.com

${ }^{2}$ Universidade de Brasília, Departamento de Geografia, LSIE, Brasília, DF, Brasil .renatofg@unb.br

${ }^{3}$ Universidade de Brasília, Departamento de Geografia, LSIE, Brasília, DF, Brasil. osmarjr@unb.br

${ }^{4}$ Universidade de Brasília, Departamento de Geografia, LSIE, Brasília, DF, Brasil. robertogomes@unb.br
} 
Sentinel-1 satellite in an urban environment. In this sense, the objective of this study was to classify the LULC of part of the Federal District, Brazil based on different dimensions considering the measures of intensity and interferometric coherence for the year 2018. The results measured from the Kappa and F1 metrics indicate that the insertion of a time series of interferometric coherencies improves the performance of the classification, from 0.50 to 0.75 (Kappa) and from 0.54 to 0.79 (F1), a fact that was evident in the improved performance of the thematic classes related to vegetation cover. Furthermore, it is also found that the identification of urban objects is best represented by the use of only the intensities (VV and VH) in the classification process.

\section{INTRODUÇÃO}

Partes significativas dos problemas sociais e ambientais são agravadas pela concentração populacional nas cidades brasileiras, visto que, aproximadamente, 160 milhões de pessoas vivam no ambiente urbano, cerca de $85 \%$ da população, conforme estimativas provenientes do Censo Demográfico de 2010 elaborado pelo Instituto Brasileiro de Geografia e Estatística (IBGE). Ademais, segundo Zhu el at. (2019), as áreas urbanas garantem mais de $75 \%$ do Produto Interno Bruto (PIB) mundial.

As informações de uso e cobertura da terra são essenciais para a elaboração de políticas públicas relacionadas ao meio ambiente (KHALIL; HAQUE, 2018). A dinâmica do uso e cobertura da terra é uma das mais expressivas áreas de estudo do sensoriamento remoto, sendo reconhecida como um elemento chave na avaliação de impactos ambientais, no monitoramento ecológico, no monitoramento das mudanças globais, no planejamento territorial estadual e local, no desenvolvimento de políticas regulatórias, bem como na identificação e prevenção da expansão irregular da malha urbana.

A evolução do imageamento SAR (Synthethic Aperture Radar) para mapeamento de uso e cobertura da terra tem demonstrado ótimos resultados por não possuir influência direta da atmosfera e da cobertura de nuvens, o que favorece a análise de mudança ao longo do tempo. Os dados obtidos por sistemas ativos vêm contribuindo e popularizando o desenvolvimento de estudos focados na classificação do uso e cobertura da terra (DINIZ; GAMA, 2019; MIGUEL; SANO, 2019), na identificação de objetos considerando a fusão com informações provenientes de sensores óticos (RAJAH et al., 2018; WERNER et al., 2014), no derramamento de óleo (CHATURVEDI et al., 2019; OZIGIS et al., 2020), nos movimentos gravitacionais de massa
(AUBLANC et al., 2018; LI et al., 2020), no mapeamento de áreas agrícolas (PICHIERRI et al., 2018; SEKERTEKIN et al., 2020), dentre outros.

Os sistemas SAR também possuem potencial na identificação de objetos urbanos. O controle quanto à radiação emitida e recebida pelo sensor, permite uma melhor compreensão das estruturas e dos objetos geométricos presentes no terreno, uma vez que suas propriedades dielétricas alteram a polarização da radiação eletromagnética (WERNER et al., 2014). Apesar disso, o sistema SAR apresenta alguns fatores que interferem na qualidade do imageamento das superfícies naturais e artificiais, devido às mudanças abruptas na altura, orientação, forma e tamanho dos objetos, que juntos promovem uma confusão espectral entre as classes de uso e cobertura da terra que serão mapeadas (GRIFFITHS et al., 2010).

A utilização da técnica interferométrica (InSAR) possibilita aprimorar as classificações de uso e cobertura da terra. A fase ondulatória (Ф) presente no sinal SAR representa o momento de retorno da onda eletromagnética e está relacionada com a distância entre a antena do satélite e os alvos, e, por isso, pode ser usada como informação na derivação de distorções no terreno (movimentos de massa, rebaixamentos, Modelos Digitais de Terreno, dentre outros). Essa informação de fase $(\Phi)$ pode ser quantificada considerando a seguinte relação matemática (Equação 1), considerando a natureza sinusoidal em relação à um círculo de 2 п.

$$
\Phi=\frac{2 \pi}{\lambda} 2 \mathrm{R}=\frac{4 \pi}{\lambda} \mathrm{R}
$$

Essa técnica é baseada na mensuração da diferença entre as fases de duas cenas SAR de uma mesma área e obtidas em ângulos diferentes, o que tipifica a mudança de fase, ou fase interferométrica $(\Delta \Phi)$. A Figura 1 esquematiza a geometria de um sistema InSAR 
obtido a partir de duas órbitas separadas por uma linha de base interferométrica.

Figura 1. Esquema da geometria InSAR obtido a partir de duas órbitas.

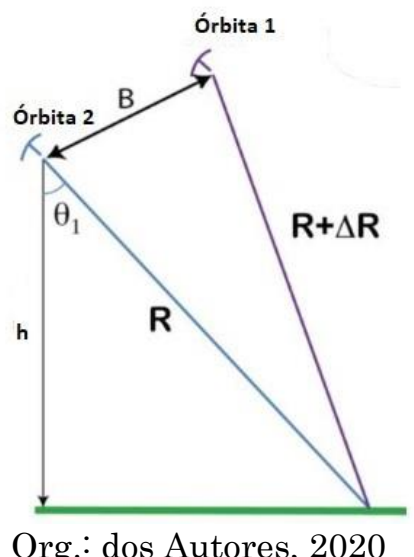

As medidas apresentadas na figura acima possuem relação direta com a distância percorrida pelo sinal, a baseline interferométrica, os ângulos de visada e o comprimento de onda (ג). As equações 2 e 3 apresentadas a seguir expressam a relação geométrica da definição conceitual do interferograma.

$$
\begin{aligned}
& \Phi_{1}=\frac{4 \pi R}{\lambda}, \Phi_{2}=\frac{4 \pi(R+\Delta R)}{\lambda} \\
& \Delta \Phi=\Phi_{2}-\Phi_{1}=\frac{4 \pi \Delta R}{\lambda}
\end{aligned}
$$

As técnicas InSAR fornecem informações que auxiliam no estudo do ambiente urbano, principalmente focadas em identificar movimentos de subsidência a partir da análise de deformações da superfície (CIAMPALINI et al., 2019; HU et al., 2019). Portanto, é fundamental garantir uma boa qualidade de ambos os sinais de retorno no sentido de proceder análises de cunho interferométrico.

A coerência (Y) é uma medida interferométrica auxiliar que varia entre 0 e 1 e indica o grau de similaridade das fases de ambos os sinais de retorno em um sistema InSAR. Essa métrica é usada para identificar as áreas passíveis de estudos interferométricos, resultado direto da combinação de determinado padrão geométrico do sistema utilizado e das condições ambientais da área imageada durante o registro de ambos os sinais. A Equação 4 define a coerência interferométrica entre duas imagens SAR, produto da relação entre os valores reais e esperados das amplitudes $\mid u_{1} /$ e $\mid u_{2} /$ das duas imagens SAR originais.

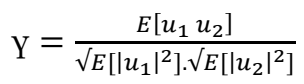

A coerência interferométrica é uma importante informação complementar para fins de classificação temática da paisagem (OLESK et al., 2016; WEGMULLER et al., 2015), uma vez que seus valores estão associados à características temporais, geométricas, volumétricas e de processamento do sinal. Tal métrica é amplamente utilizada em estudos que envolvem a identificação da cobertura vegetal (DINIZ; GAMA, 2019; MOHAMMADIMANESH et al., 2018), devido seu característico comportamento volumétrico em série temporal. Tais áreas são bastante heterogêneas e apresentam elevadas mudanças em textura ao longo do tempo, fatos ocasionados pelas diferentes condições climáticas ou mesmo pela conversão de uso da terra. A variação dos valores de coerência ocasionada pela decorrelação temporal auxilia na identificação de diferentes curvas de comportamento espectro-temporal desses alvos e servem de informação complementar para sua classificação.

A coerência interferométrica também possui um elevado potencial de aplicação no mapeamento do uso e ocupação da terra em ambiente urbano (MIGUEL; SANO, 2019; SICA et al., 2019; ZHANG et al., 2018). De forma geral, os objetos urbanos (prédios, casas, muros, dentre outros) não possuem uma grande variação de valores de retroespalhamento considerando uma série temporal, o que ocasiona o registro de altos índices de coerência sobre tais áreas. Ademais, limitados estudos foram realizados utilizando-se a coerência interferométrica proveniente do satélite Sentinel-1 em ambiente urbano (KHALIL; HAQUE, 2018).

Desta forma, o objetivo desse estudo é classificar o uso e cobertura da terra de parte do Distrito Federal utilizando-se informações provenientes de série temporal composta de imagens SAR do satélite Sentinel 1 relativas ao ano de 2018. Para tanto, diferentes arranjos temporais considerando as medidas de intensidade do sinal (db) e da coerência interferométrica foram testados visando identificar a real contribuição de tais métricas no processo de classificação. 


\section{ÁREA DE ESTUDO}

O Distrito Federal (DF) possui uma população de, aproximadamente, 3.000.000 habitantes, que se distribui de forma assimétrica entre o ambiente rural (3,38\%) e urbano (96,62\%), conforme consta em seu Zoneamento EcológicoEconômico (BRASIL, 2018). O ambiente urbano do Distrito Federal possui diversos arranjos que se materializam, principalmente, em sua porção planejada, com prédios e residências dispostas em áreas arborizadas, variando em densidade, ou não-planejada, no qual tendem a se comportar como arranjos típicos urbanos encontrados em outras cidades brasileiras. A Figura 2 ilustra diferentes arranjos de objetos urbanos que podem ser encontrados no Distrito Federal.
Assim, a área de estudo localiza-se em parte do DF e compreende o perímetro estabelecido entre as coordenadas 48.5'57,31"O $15^{\circ} 34^{\prime} 23,04^{\prime \prime}$ S e $47^{\circ} 49^{\prime} 50,10^{\prime \prime O} 15^{\circ} 57^{\prime} 8,3^{\prime \prime}$ S. A escolha dessa área está relacionada (i) a diversificação existente de morfologias urbanas e de cobertura vegetal associada à fitofisionomia de cerrado e (ii) ao limite tecnológico existente, à época de processamento dos dados, relacionado a capacidade computacional disponível e a grande quantidade de dados SAR processados relativos a todo o ano de 2018, especialmente os interferométricos. A Figura 3 mostra a porção do Distrito Federal que foi utilizada nas inferências espaciais realizadas pela presente pesquisa.

Figura 2. Diferentes arranjos urbanos no Distrito Federal. (a) Área planejada e amplamente arborizada; (b) Área planejada e menos arborizada; (c) Área de expansão não-planejada recente

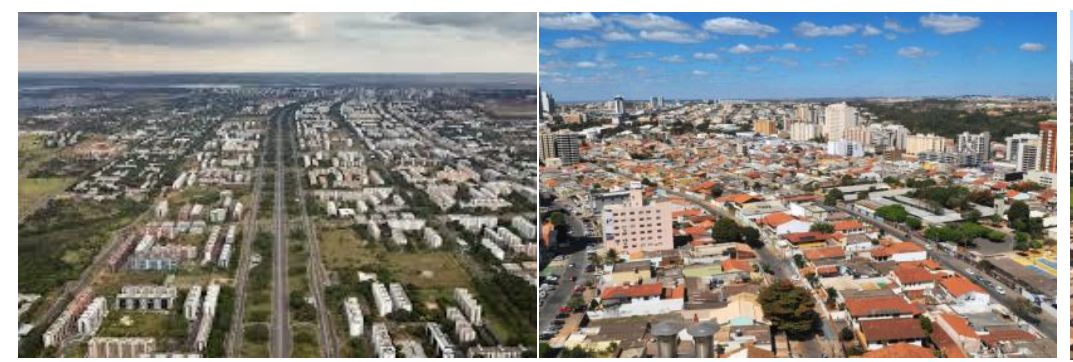

(a) (b)

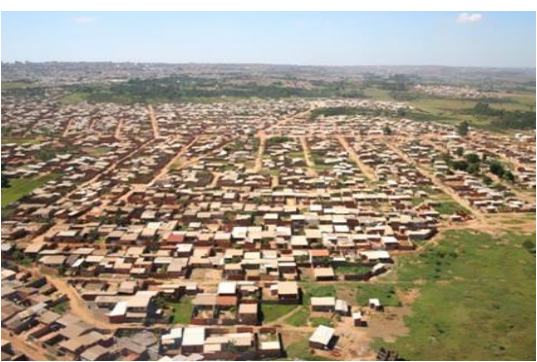

(c)

Org.: dos Autores, 2020

Figura 3. Área de estudo referente à porção do Distrito Federal

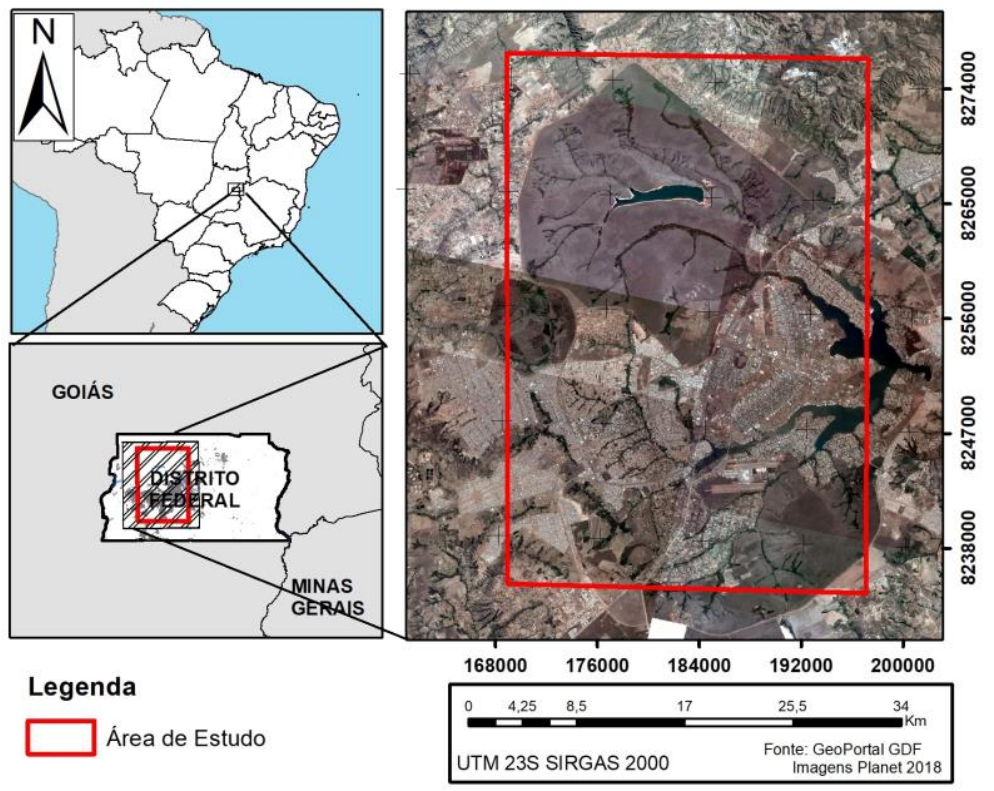

Org.: dos Autores, 2020. 


\section{MATERIAL E MÉTODOS}

A metodologia do trabalho foi organizada em 3 etapas (Figura 4). A primeira etapa refere-se aos pré-processamentos dos sinais de radar e a construção dos cubos temporais. A segunda etapa refere-se à definição das classes temáticas e a escolha das amostras de treinamento. A terceira etapa refere-se aos procedimentos de classificação e de avaliação dos resultados.

Figura 4. Fluxograma das etapas metodológicas do trabalho.

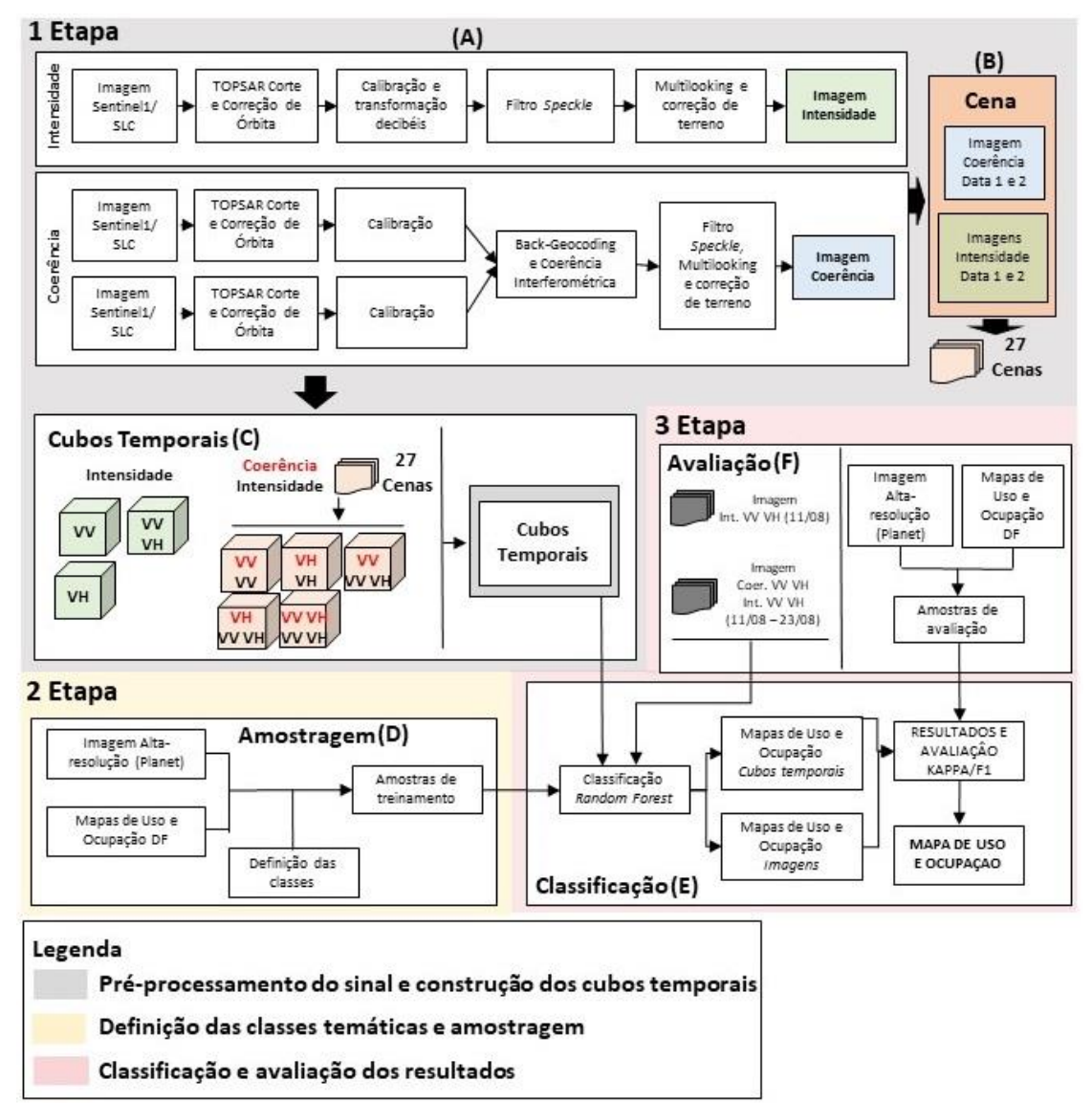

Org: dos Autores, 2020

Pré-processamento do sinal de radar e construção dos cubos temporais (Etapa 1)

O Sentinel 1, iniciativa da Comissão Europeia e da Agência Espacial Europeia (ESA), é uma constelação de duas plataformas orbitais operando na banda C $(5.405 \mathrm{GHz})$ do microondas, Sentinel 1A e Sentinel 1B, em duas polarizações (VV e VH), e conta com 4 (quatro) modos de aquisição: (i) Stripmap (SM); (ii) Interferometric Wide Swath (IW); (iii) ExtraWide $S_{\text {wath }}(\mathrm{EW})$; e (iv) Wave mode (WV). O modo de aquisição definido foi o IW, que apresenta uma faixa de imageamento de 250 $\mathrm{km}$ com uma resolução geométrica moderada $(5 \mathrm{~m}$ por $20 \mathrm{~m})$ e estruturada em Terrain Observation with Progressive Scans SAR (TOPSAR), que garante uma qualidade homogênea na imagem e satisfaz a maioria das aplicações em terreno (DE ZAN; GUARNIERI, 2006). Esse modo de aquisição é o mais utilizado para mapeamentos de uso e ocupação da terra.

As imagens do satélite Sentinel 1 podem ser adquiridas em diferentes níveis de processamento, a saber: (i) Single Look Complex (SLC); e (ii) Ground Range Detected (GRD). Tendo em perspectiva a aplicação interferométrica, foram selecionadas imagens em nível de processamento SLC, relativas ao ano de 2018, uma vez que possuem informações relativas a amplitude e a fase do sinal, registradas em números complexos, e projetadas na geometria de visada do sensor (slant-range). A Tabela 1 mostra as datas das 28 imagens Sentinel 1 IW/SLC que foram adquiridas. 
Tabela 1. Datas das imagens Sentinel 1 adquiridas e processadas.

\begin{tabular}{lccc}
\hline \multicolumn{4}{c}{ Datas - Imagens Sentinel 1 (IW SLC) } \\
$07 / 01 / 2018$ & $01 / 04 / 2018$ & $18 / 07 / 2018$ & $10 / 10 / 2018$ \\
$19 / 01 / 2018$ & $13 / 04 / 2018$ & $30 / 07 / 2018$ & $22 / 10 / 2018$ \\
$31 / 01 / 2018$ & $25 / 04 / 2018$ & $11 / 08 / 2018$ & $03 / 11 / 2018$ \\
$12 / 02 / 2018$ & $07 / 05 / 2018$ & $23 / 08 / 2018$ & $15 / 11 / 2018$ \\
$24 / 02 / 2018$ & $19 / 05 / 2018$ & $04 / 09 / 2018$ & $27 / 11 / 2018$ \\
$08 / 03 / 2018$ & $12 / 06 / 2018$ & $16 / 09 / 2018$ & $09 / 12 / 2018$ \\
$20 / 03 / 2018$ & $24 / 06 / 2018$ & $28 / 09 / 2018$ & $21 / 12 / 2018$ \\
\hline
\end{tabular}

Org.: dos Autores, 2020.

A etapa de pré-processamento do sinal de radar (Figura 4A) tem por objetivo reduzir as distorções radiométricas e geométricas e corrigir as imagens SAR. Essa etapa foi realizada considerando as aplicações presentes no programa SNAP, desenvolvido pela Agência Espacial Europeia (ESA), que possui ferramentas específicas para o processamento e a estimativa das métricas de intensidade e de coerência interferométrica do satélite Sentinel 1.

Todas as imagens SAR foram recortadas para a área de interesse e corrigidas considerando suas informações complementares relativas à órbita e a altitude do satélite. No caso da estimativa dos valores de intensidade, o sinal foi calibrado com o objetivo de realizar a transformação dos valores de retroespalhamento, inicialmente, em níveis digitais (ND) para sigma ( $\sigma$ ) e, posteriormente, transformado em níveis de decibéis (db), etapa essa que garante uma melhor representação dos valores no intervalo do histograma da imagem, a partir de sua expansão.

Após os ajustes radiométricos, foi necessária a realização de um procedimento de filtragem convolucional, utilizando-se a mediana em uma janela $5 \times 5$, no sentido de reduzir o efeito speckle inerente a dados SAR. Esse efeito provoca uma diferenciação nos valores de retroespalhamento que podem não estar associados a mudanças reais de objetos na paisagem, causando um padrão granulado do tipo "sal e pimenta". Posteriormente, realizouse a transformação das imagens, originalmente, projetada slant-range para uma grade regular, processo conhecido como multilooking.

Por fim, as imagens foram ortorretificadas e projetadas em UTM, fuso 23S, e datum WGS 1984, tendo como referência o Modelo Digital de Elevação do Shuttle Radar Topography Mission (SRTM) de 30 metros de resolução espacial, o que resultaram em imagens SAR finais na resolução espacial de 10 metros. Tais correções geométricas de terreno visam reduzir as distorções associadas ao encurtamento de rampa, dentre outros.

Para a estimativa da coerência, o sinal SAR também foi recortado para a área de interesse e calibrado mantendo-se as informações de amplitude e fase, fato que demanda a manutenção dos valores ajustados em números complexos. Como a configuração de um sistema InSAR com imagens Sentinel 1 só é possível de forma monostática, no qual a simulação da linha de base interferométrica é adquirida com a aquisição de duas cenas de tempos distintos sobre uma mesma área, pares de imagens foram agrupados considerando as informações complementares de órbita através do processo back-geocoding, visando georeferenciar as informações de amplitude e fase e, assim, permitir o computo da coerência interferométrica conforme a Equação 4. O Quadro 1 mostra as 27 imagens de coerência interferométrica calculadas para o ano de 2018.

Uma vez calculadas as coerências interferométricas apresentadas no quadro 1 , os procedimentos de filtragem, multilooking e correção geométrica de terreno adotados foram os mesmos já apresentados acima. Isso se justifica uma vez que os valores de coerência interferométrica são computados na projeção slant-range utilizando-se as informações de fase do sinal original, registrado em números complexos, em cada imagem do par, visando à compatibilidade com as métricas de intensidade. As imagens de coerência interferométrica resultantes também foram elaboradas na resolução espacial de 10 metros. Todas as informações SAR processadas foram transformadas para SIRGAS 2000, observando a Resolução IBGE 01/2015 (BRASIL, 2015). 
Quadro 1. Arranjo adotado para a estimativa das coerência interferométrica de 2018.

\begin{tabular}{|c|c|c|c|c|c|c|c|}
\hline Data & Coer. & Data & Coer. & Data & Coer. & Data & Coer \\
\hline $07 / 01 / 2018$ & \multirow{2}{*}{1} & $01 / 04 / 2018$ & \multirow{2}{*}{8} & $18 / 07 / 2018$ & \multirow{2}{*}{15} & $10 / 10 / 2018$ & \multirow{2}{*}{22} \\
\hline $19 / 01 / 2018$ & & $13 / 04 / 2018$ & & $30 / 07 / 2018$ & & $22 / 10 / 2018$ & \\
\hline $19 / 01 / 2018$ & \multirow{2}{*}{2} & $13 / 04 / 2018$ & \multirow{2}{*}{9} & $30 / 07 / 2018$ & \multirow{2}{*}{16} & $22 / 10 / 2018$ & \multirow{2}{*}{23} \\
\hline $31 / 01 / 2018$ & & $25 / 04 / 2018$ & & $11 / 08 / 2018$ & & 03/11/2018 & \\
\hline $31 / 01 / 2018$ & \multirow{2}{*}{3} & $25 / 04 / 2018$ & \multirow[b]{2}{*}{10} & $11 / 08 / 2018$ & \multirow[b]{2}{*}{17} & $03 / 11 / 2018$ & \multirow[b]{2}{*}{24} \\
\hline $12 / 02 / 2018$ & & $07 / 05 / 2018$ & & $23 / 08 / 2018$ & & $15 / 11 / 2018$ & \\
\hline $12 / 02 / 2018$ & \multirow{2}{*}{4} & $07 / 05 / 2018$ & \multirow{2}{*}{11} & $23 / 08 / 2018$ & \multirow{2}{*}{18} & $15 / 11 / 2018$ & \multirow{2}{*}{25} \\
\hline $24 / 02 / 2018$ & & $19 / 05 / 2018$ & & $04 / 09 / 2018$ & & $27 / 11 / 2018$ & \\
\hline $24 / 02 / 2018$ & \multirow{2}{*}{5} & $19 / 05 / 2018$ & \multirow{2}{*}{12} & $04 / 09 / 2018$ & \multirow{2}{*}{19} & $27 / 11 / 2018$ & \multirow{2}{*}{26} \\
\hline $08 / 03 / 2018$ & & $12 / 06 / 2018$ & & $16 / 09 / 2018$ & & $09 / 12 / 2018$ & \\
\hline $08 / 03 / 2018$ & \multirow[b]{2}{*}{6} & $12 / 06 / 2018$ & \multirow[b]{2}{*}{13} & $16 / 09 / 2018$ & \multirow[b]{2}{*}{20} & $09 / 12 / 2018$ & \multirow[b]{2}{*}{27} \\
\hline $20 / 03 / 2018$ & & $24 / 06 / 2018$ & & $28 / 09 / 2018$ & & $21 / 12 / 2018$ & \\
\hline $20 / 03 / 2018$ & \multirow[b]{2}{*}{7} & $24 / 06 / 2018$ & \multirow{2}{*}{14} & $28 / 09 / 2018$ & \multirow{2}{*}{21} & & \\
\hline $01 / 04 / 2018$ & & $18 / 07 / 2018$ & & $10 / 10 / 2018$ & & & \\
\hline
\end{tabular}

Org.: dos Autores, 2020

Uma vez pré-processadas as informações, as imagens de intensidade relativas ao ano de 2018 foram combinadas em diferentes estruturas matriciais contendo toda a informação do interstício analisado (cubos temporais), conforme apresentado na Figura 4C. Esse arranjo é específico para a análise espectro-temporal no âmbito do programa Abilius, que possui capacidade de análise quantitativa de um alvo considerando sua variabilidade temporal.

Diferentes combinações de cubos temporais foram estruturados considerando, inicialmente, somente as métricas de intensidade em ambas as polarizações, cuja intenção foi verificar o desempenho no processo de classificação considerando as possíveis combinações das polarizações disponíveis. Ademais, e visando compatibilizar as informações das coerências interferométricas e das intensidades em uma mesma estrutura matricial, foi necessário, inicialmente, estabelecer 27 cenas distintas, cada qual com uma (i) imagem de coerência e (ii) com o valor médio das intensidades relativas as datas das imagens que formaram o respectivo par interferométrico (Figura 4B), que, por fim, foram agrupadas observando-se os arranjos temporais já mencionados (Quadro 1).

Assim, foi possível estruturar 3 cubos temporais utilizando-se apenas as medidas de intensidade, e 5 cubos temporais considerando as possíveis combinações entre as medidas de coerência interferométrica e de intensidade, em suas diferentes polarizações (Figura 4C). Esse arranjo metodológico permitiu a aquisição de 8 classificações de uso e ocupação da terra para a série temporal de 2018.

\section{Definição das classes e das amostras de treinamento (Etapa 2)}

A pesquisa utilizou 7 classes temáticas para o mapeamento do uso e cobertura da terra, sendo elas: (i) água; (ii) cobertura vegetal - formação campestre; (iii) cobertura vegetal - formação savânica; (iv) cobertura vegetal - formação florestal; (v) áreas planas, (vi) mancha urbana e (vii) edificações (Figura 4D). As quatro primeiras classes são comumente utilizadas em mapas de uso e ocupação da terra e serviram para registrar as localidades com presença de água e os diferentes arranjos de cobertura vegetal. Por sua vez, a classe "áreas planas" tem por objetivo registrar as localidades que apresentam baixos valores de intensidade, uma vez que são planas em relação às configurações do sistema SAR utilizado, tais como pistas, estacionamentos e solo exposto. Essa classe foi primordial no sentido de evitar possíveis confusões com a classe de água, uma vez que as respostas do retroespalhamento do sinal são muito similares devido a presença dominante do efeito especular.

A temática urbana utilizada nesse trabalho foi estruturada considerando dois focos: (i) a identificação de áreas contendo o agregado de objetos urbanos (edifícios e construções) e áreas verdes; e (ii) a identificação dos próprios objetos urbanos. Essas áreas possuem dupla ou tripla reflexão do sinal em resultado a sua interação com objetos que possuem superfícies planas e 
ortogonais inerentes a fachadas de prédios, denominado de efeito de refletor de canto (double-bounce), o que favorece a identificação de tais alvos. Para o treinamento da primeira vertente, a classe temática "mancha urbana" foi definida considerando diferentes arranjos quanto à orientação e concentração de objetos urbanos, bem como com relação às áreas verdes circunvizinhas (Figura 2). Assim, essa classe temática teve o intuito de servir de transição entre os objetos urbanos e as demais classes presentes no mapeamento, porque o termo "urbano" não possui um sentido único e torna impossível a definição exata de diferentes estruturas físicas e suas propriedades de superfície. A segunda vertente consistiu do treinamento de amostras relativas às construções mapeadas por intermédio do aerolevantamento cadastral do DF realizado em 2016.

Para cada uma das classes temáticas foram estabelecidas 20 regiões de interesse, que representaram um agrupamento amostral de, aproximadamente, 6.400 pixels. $^{O}$ intuito dessa estratégia adotada foi manter a distribuição estratiforme entre as amostras em cada um dos processos de classificação. Esse processo de amostragem contou com o auxílio: (i) de fotointerpretação de imagens do satélite ótico de alta resolução Planet do ano de 2018; (ii) mapas de vegetação do Distrito Federal elaborados pelo governo local no âmbito do Zoneamento Ecológico-Econômico do Distrito Federal (BRASIL, 2018); e (iii) mapa de uso e cobertura da terra elaborado em 2019 pelo governo local.

A única exceção foi com relação à classe "edificações", que foi treinada contando com a seleção aleatória de 100 construções, com mais de $500 \mathrm{~m}^{2}$ cada, proveniente das informações cadastrais urbanas do aerolevantamento realizado em 2016. Esses quantitativos foram definidos visando garantir o mesmo valor de agrupamento amostral das demais classes temáticas de treinamento. Essa etapa foi realizada com o auxílio do programa ENVI 4.3.

\section{Etapa de classificação (Etapa 3 E)}

$\mathrm{O}$ algoritmo Random Forest (RF) foi, inicialmente, desenvolvido por Breiman (2001) e consiste de um processo de predição de resultados tendo por base o desenvolvimento e a utilização de um conjunto de árvores de decisão criadas aleatoriamente com as informações relevantes para a classificação e são estruturadas tendo por base o melhor arranjo de decisão para a explicação de determinada informação. Para cada kth árvore do conjunto a ser constituído pelo algoritmo, um vetor aleatório $\odot_{k}$ é gerado, independente dos vetores aleatórios passados $\odot_{1}, \ldots, \odot_{k^{-1}}$, mas com a mesma distribuição. Assim, uma árvore é estruturada utilizando-se as amostras de treinamento e $\Theta_{k}$, resultando na definição do classificador $h\left(\mathrm{x}, \odot_{k}\right)$. Os nós de cada uma das $k$ th árvore são definidos tomando-se por base a natureza dos dados de entrada de forma aleatória, buscando-se sempre garantir a melhor entropia entre um nó e seu subsequente (BELGIU; DRAGUT, 2016; BREIMAN, 2001; WHITCOMB et al, 2009). Após a estruturação de um número elevado de árvores, cada classificador estabelecido é utilizado no processo de votação do algoritmo pela classe com maior probabilidade de ocorrência.

A escolha desse classificador se justifica uma vez que quando as amostras de treinamento são menores, ou mesmo o padrão de qualidade é desconhecido, o algoritmo mais robusto a ser utilizado nessa situação são os associados à conjunto de árvores de decisão (MAXWELL et al., 2018). Resultados satisfatórios referentes à potencialidade do $\mathrm{RF}$ frente aos demais algoritmos de machine learning foram encontrados nos trabalhos de Miguel e Sano (2019) e Lawrence e Moran (2015).

O programa livre Abilius vem sendo desenvolvido no Laboratório de Sistema de Informações Espaciais (LSIE) da Universidade de Brasília (UnB) e possui ferramentas de visualização e processamento digital de imagens e algoritmos de classificação machine learning. A principal vantagem da escolha desse software está em sua capacidade de análise em série temporal, no qual as informações são estruturadas em cubos temporais visando permitir a análise quantitativa da dinâmica espectro-temporal dos diferentes alvos da imagem.

O algoritmo RF implementado no Abilius é proveniente da biblioteca Open Source Vision Library (OpenCV) e sua parametrização foi estabelecida conforme Prinzie e Van den Poel (2008). Neste sentido, foi definido em 5 o número máximo de divisão das árvores de decisão e em 500 o número máximo do conjunto de árvores (MAXWELL et al., 2018). Esse algoritmo de classificação já foi utilizado em diversos trabalhos acadêmicos que envolveram a classificação de commodities agrícolas (BITENCOURT, 2020; COSTA, 2020), do uso e ocupação da terra (MARQUES et al., 2019; 
SALGADO, 2019), identificação de áreas inundáveis (FERREIRA, 2018) e de queimada (OLIVEIRA, 2019).

\section{Avaliação das classificações (Etapa 3 F)}

A etapa de avaliação foi estabelecida tendo por base a comparação da sequência de classificações temporais junto a um conjunto independente de dados "verdade", no sentido de viabilizar o cálculo das seguintes métricas de acurária: (i) índice Kappa e (ii) índice F1, amplamente utilizadas em estudos de sensoriamento remoto (CAO et al., 2020; SALGADO et al., 2019).

Tal como o índice Kappa, o cálculo da métrica F1 também parte de uma análise que utiliza a distribuição dos elementos da matriz de confusão, contabilizando os verdadeiros positivos (TP), os falsos positivos (FP), os verdadeiros negativos (TN) e os falsos negativos (FN). Para a estimativa de F1 (Equação 5), é necessário a estimativa do precision (Equação 6) e do recall (Equação 7), métricas complementares que indicam, respectivamente, a precisão do modelo e sua capacidade de representar a classe pretendida.

$$
\begin{aligned}
& \text { Precision }=\frac{\Sigma \text { Verdadeiros positivos }(T P)}{\Sigma \text { Verdadeiros positivos }(T P)+\Sigma F a l s o s \text { positivos }(F P)} \\
& \text { Recall }=\frac{\Sigma \text { Verdadeiros positivos }(\mathrm{TP})}{\Sigma \text { Verdadeiros positivos }(\mathrm{TP})+\Sigma \text { Falsos negativos }(\mathrm{FN})} \\
& \mathrm{F} 1=2 * \frac{\text { Precision } * \text { Recall }}{\text { Precision }+ \text { Recall }}
\end{aligned}
$$

Um resultado que apresente um alto valor de precision e um baixo valor de recall indica que poucas áreas foram devidamente mapeadas para determinada classe temática, mesmo que muitas dessas estejam corretas ao se comparar com a "verdade". Por sua vez, valores altos de recall e baixos valores de precision indicam que a classe temática foi amplamente classificada e generalizada na área de estudo, contendo muitos erros de comissão. Para cada uma das 7 classes temáticas foram estimados 300 pontos aleatórios de "verdade" independentes das amostras de treinamento (BITENCOURT, 2020; COSTA, 2020). Também para a definição desses pontos aleatórios utilizou-se o mesmo elenco de produtos cartográficos para a definição das classes e das amostras de treinamento (Etapa 2).

Por fim, e com o objetivo de avaliar o desempenho da análise em série temporal, os resultados classificatórios também foram comparados com classificações realizadas apenas com 2 imagens. A primeira foi estruturada apenas com as métricas de intensidade nas polarizações $\mathrm{VV}$ e $\mathrm{VH}$ de 11/08/2018. A segunda foi uma imagem contendo as métricas de coerência interferométrica e de intensidade, em ambas as polarizações, referentes às datas de 11/08/2018 e 23/08/2018. Essas etapas estão inseridas na Figura 4F.

\section{Consolidação do mapa de uso e ocupação da terra (Etapa 3 E)}

A consolidação final do mapa de uso e ocupação da terra é resultado do agrupamento espacial das classes temáticas mapeadas considerando seus melhores desempenhos alcançados nos processos de classificação.

Considerando que a classe temática "edificações" teve sua melhor definição em resultado classificatório diferente das demais classes, sua inserção ao mapeamento final foi realizada conforme o agrupamento espacial apresentada na Figura 5, através da aplicação de matrizes de mapas. 
Figura 5. Fluxograma metodológico de consolidação do mapa de uso e ocupação da terra

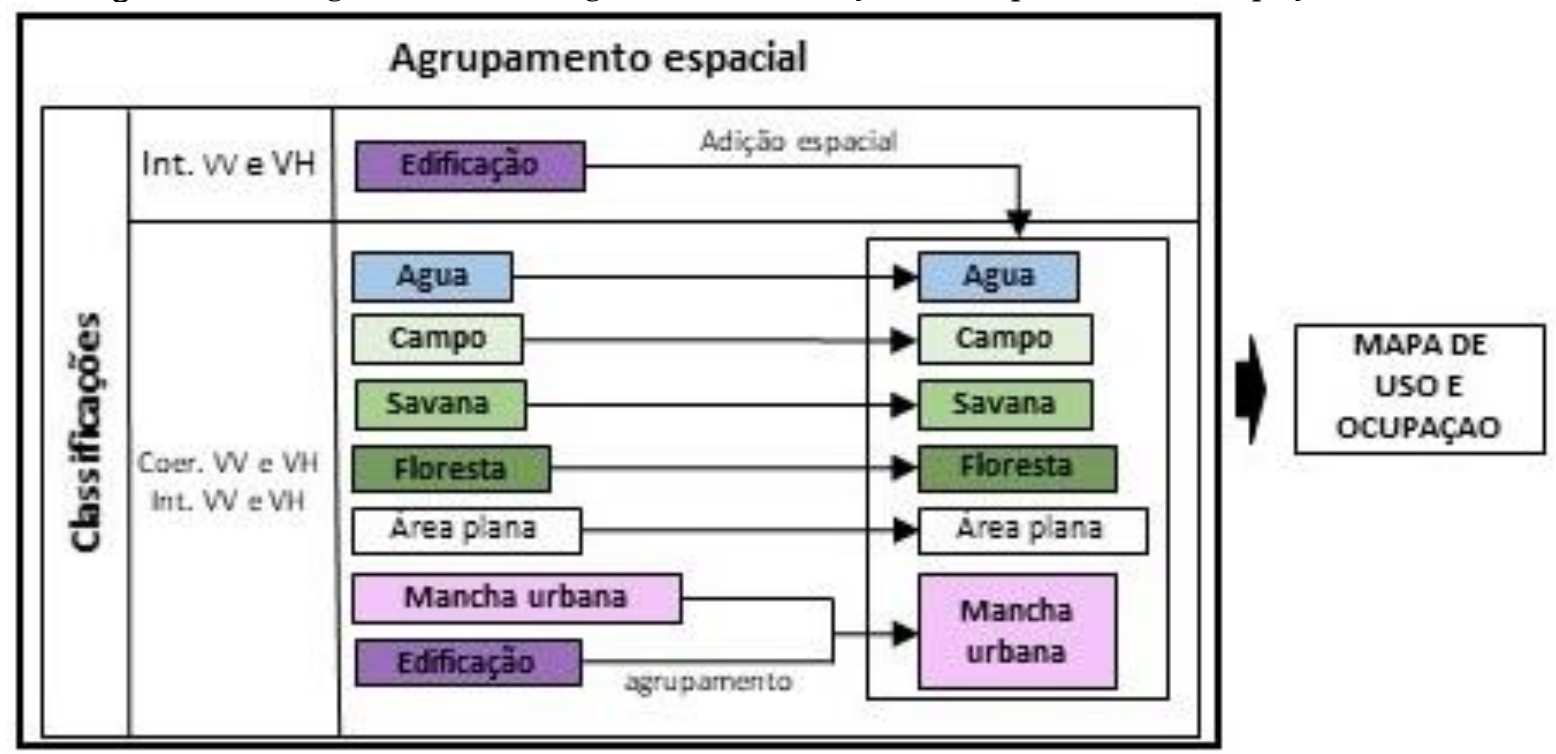

Org.: dos Autores, 2020

\section{RESULTADOS E DISCUSSÃO}

Essa seção apresenta os resultados e discussões referentes as 8 diferentes classificações temáticas realizadas sobre os cubos temporais. Nas Figuras 6 e 7 são mostrados os resultados das métricas de acurácia relativas ao índice Kappa e ao índice F1, respectivamente, entre as classificações que apresentaram melhor desempenho, tanto considerando (i) apenas a intensidade (VV e VH) (cor marrom) e (ii) a conjugação com a coerência interferométrica (cor azul), e (iii) as classificações resultantes das análises de imagens de 11/08 e 23/08. Os resultados demonstram que as análises em série temporal produzem uma considerável melhoria na classificação, o que representa um aumento de 0,42 a 0,56 (Kappa) e 0,49 a 0,59 (F1) na classificação contendo apenas as intensidades em $\mathrm{VV}$ e $\mathrm{VH}$, e de 0,6 a 0,75
(Kappa) e 0,65 a 0,79 (F1) na classificação contando a conjugação com a coerência interferométrica. O cubo temporal, com as combinações de coerência e intensidade utilizando ambas polarizações, foi o que alcançou os melhores desempenhos com 0,75 (Kappa) e 0,79 (F1). Ademais, os resultados também indicam melhores desempenhos das classificações provenientes da série temporal se comparado aos resultados de um único período.

A Figura 8 apresenta os resultados referentes ao índice F1 para cada uma das classes temáticas do trabalho, uma vez que se observaram comportamentos distintos na identificação dos alvos com relação à inserção da coerência interferométrica nas análises classificatórias. A Figura 9 apresenta as métricas precision e recall para cada uma das classes temáticas do trabalho. 
Figura 6. Desempenho das classificações a partir do índice Kappa

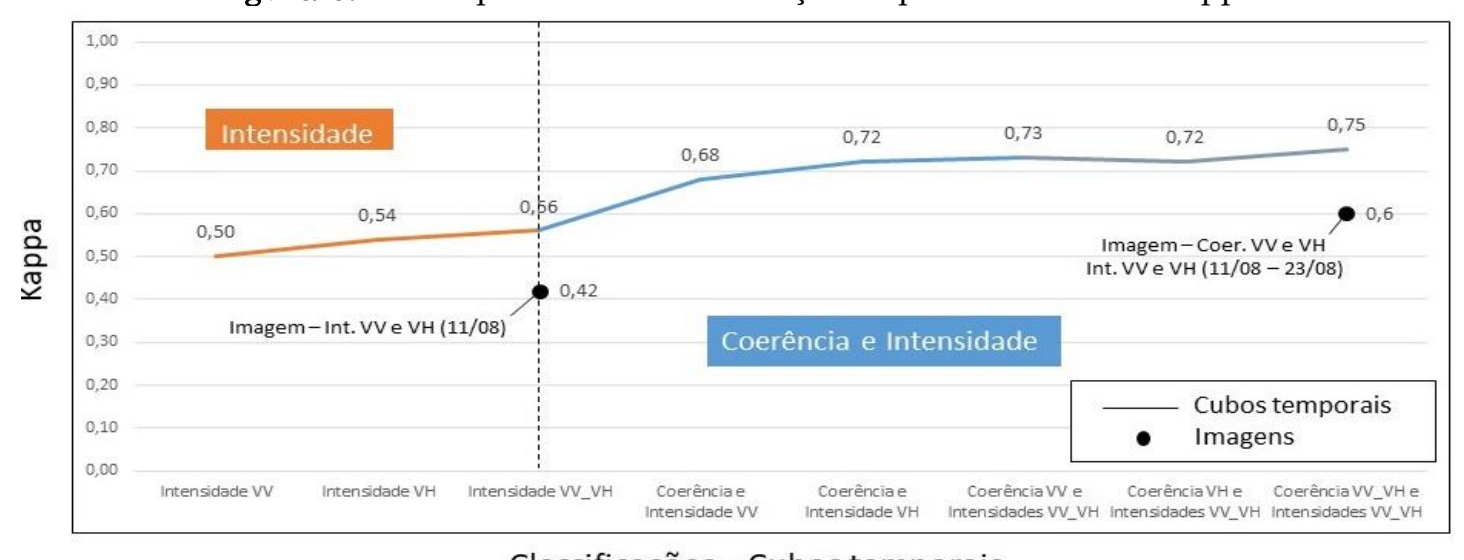

Classificações - Cubos temporais

Org.: dos Autores, 2020

Figura 7. Desempenho das classificações - índice F1, precision e recall

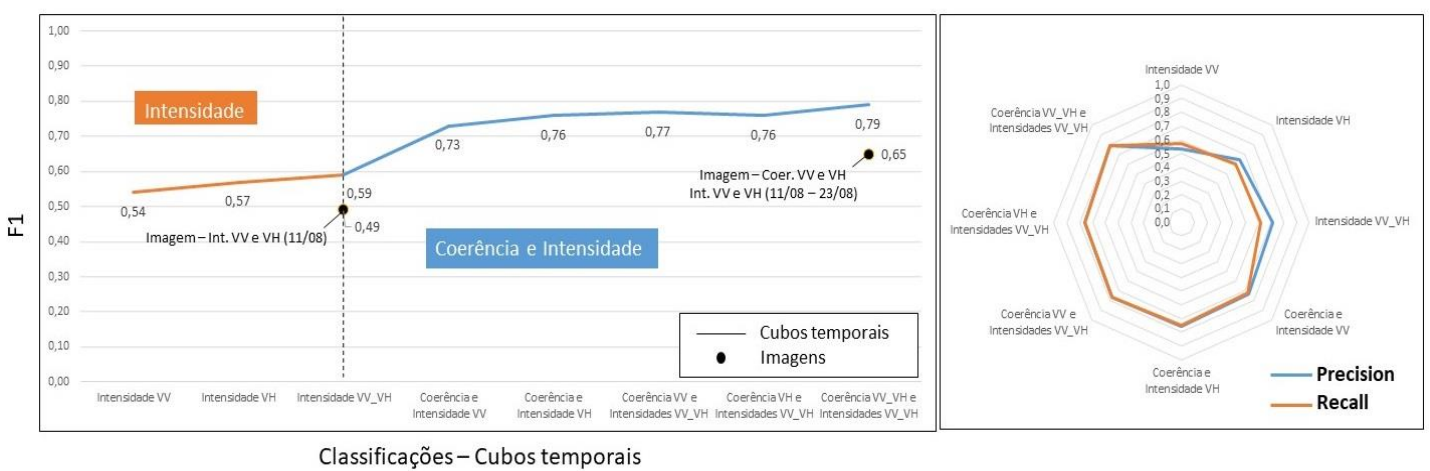

Org:: dos Autores, 2020

Em linhas gerais, constata-se que os melhores valores de F1 são alcançados pela classificação 8 que considera as intensidades e a coerência interferométrica em $\mathrm{VV}$ e $\mathrm{VH}$, fato corroborado pelo melhor balanceamento dos valores de precision e recall para essa classificação (Figura 9).

Ademais, observa-se que os valores de F1 apresentam alterações significativas no desempenho das classes temáticas relacionadas à cobertura vegetal, especificamente nas relativas à formação savânica e florestal (Figura 8c e 8d). Os resultados indicam um incremento de 0,51 a 0,70 (savana) e de 0,47 a 0,93 (floresta) ao se considerar a coerência interferométrica na classificação, no caso, considerando todas as possíveis combinações das polarizações.
Conforme observa-se na Figura 9c, a classe "savana" apresentou pouca variação dos valores de recall e uma melhora significativa dos valores de precision $(0,39$ a 0,63$)$, o que indica uma diminuição dos falsos positivos. Por sua vez, na classe "floresta" (Figura 9d) a melhora de desempenho fica mais perceptível, no qual ambas as métricas precision e recall aumentaram, respectivamente, de 0,46 a 0,90 e de 0,49 a 0,96 . Esses resultados indicam uma diminuição significativa dos falsos negativos e positivos, e garante, assim, a definição de um modelo mais fidedigno na identificação de classes vegetacionais, principalmente aquelas que possuem menor variação temporal em sua densidade florestal. A Figura 10 apresenta a melhora no desempenho da classificação das classes de cobertura vegetal com a inserção da coerência interferométrica em série temporal. 
Figura 8. Desempenho das classificações por classe temática a partir do índice F1 - (a) Água; (b)

Formação campestre; (c) Formação savânica; (d) Formação florestal; (e) Mancha urbana; (f) Edificação e (g) Áreas planas

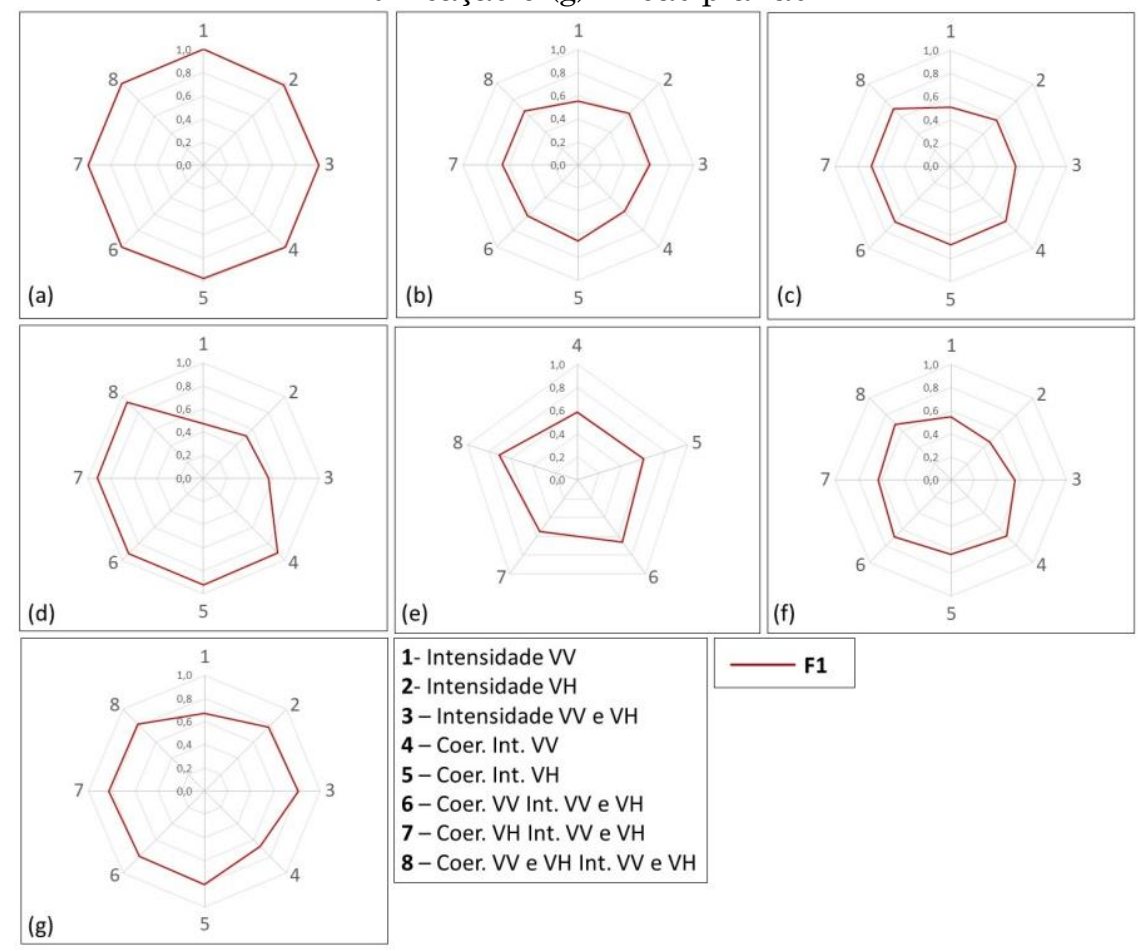

Org.: dos Autores, 2020

Figura 9. Desempenho das classificações por classe temática - índice precision e recall - (a) Água; (b) Formação campestre; (c) Formação savânica; (d) Formação florestal; (e) Mancha urbana; (f)

Edificação e (g) Áreas planas

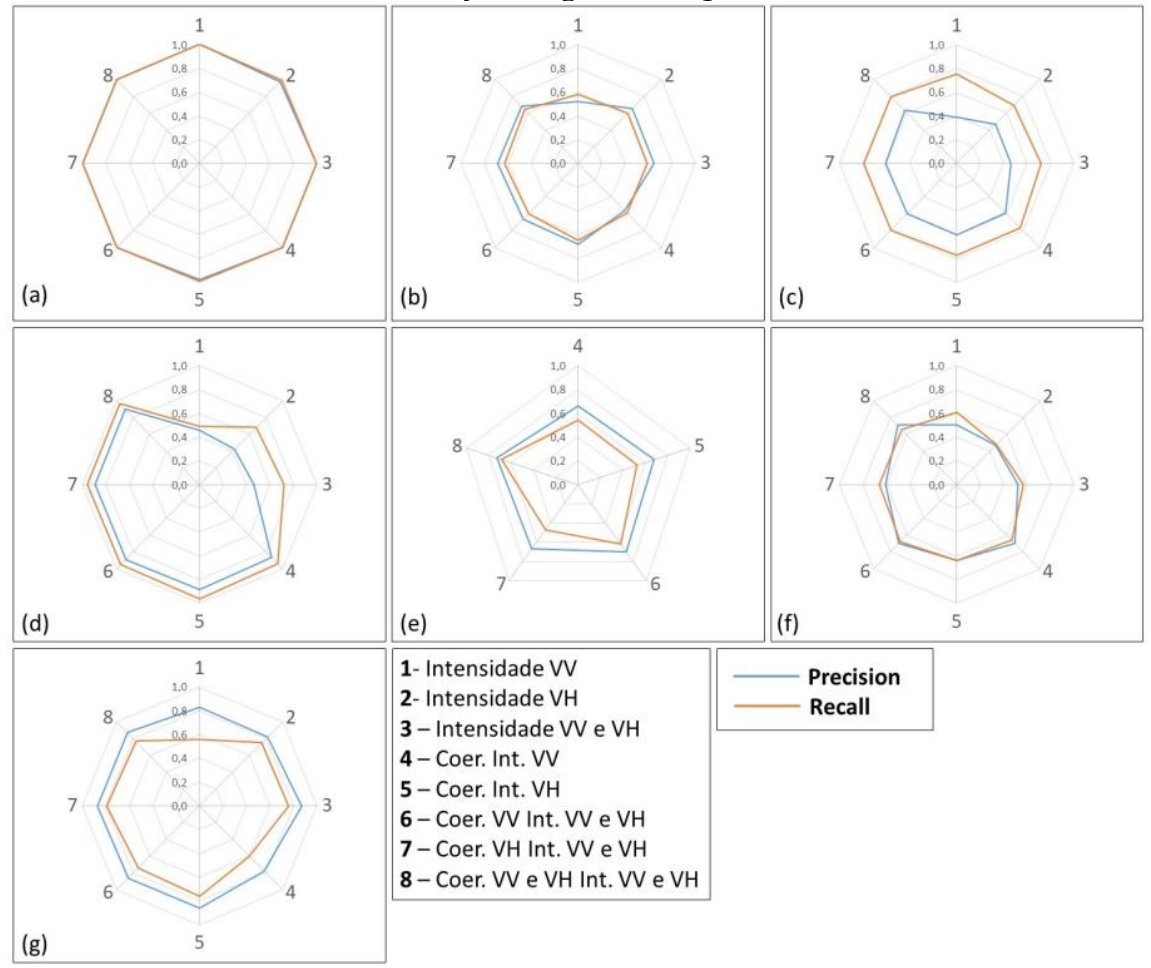

Org.: dos Autores, 2020. 


\section{Objetos urbanos versus mancha urbana}

As classes temáticas relativas ao ambiente urbano apresentaram comportamentos distinto com a inserção da coerência no processo classificatório. A classe "mancha urbana" somente se materializou como resultado nas classificações quando a coerência foi inserida na dimensionalidade dos dados analisados, mesmo que seu treinamento tenha sido realizado observando o método adotado. Conforme consta na Figura 8e, percebe-se um incremento de 0,59 a 0,71 nos valores de $\mathrm{F} 1$ ao se utilizar as intensidades e as coerências em ambas as polarizações no processo de classificação, bem como um melhor balanceamento entre os valores de precision e recall (Figura 9e).

Figura 10. Desempenho das classes de cobertura vegetal.

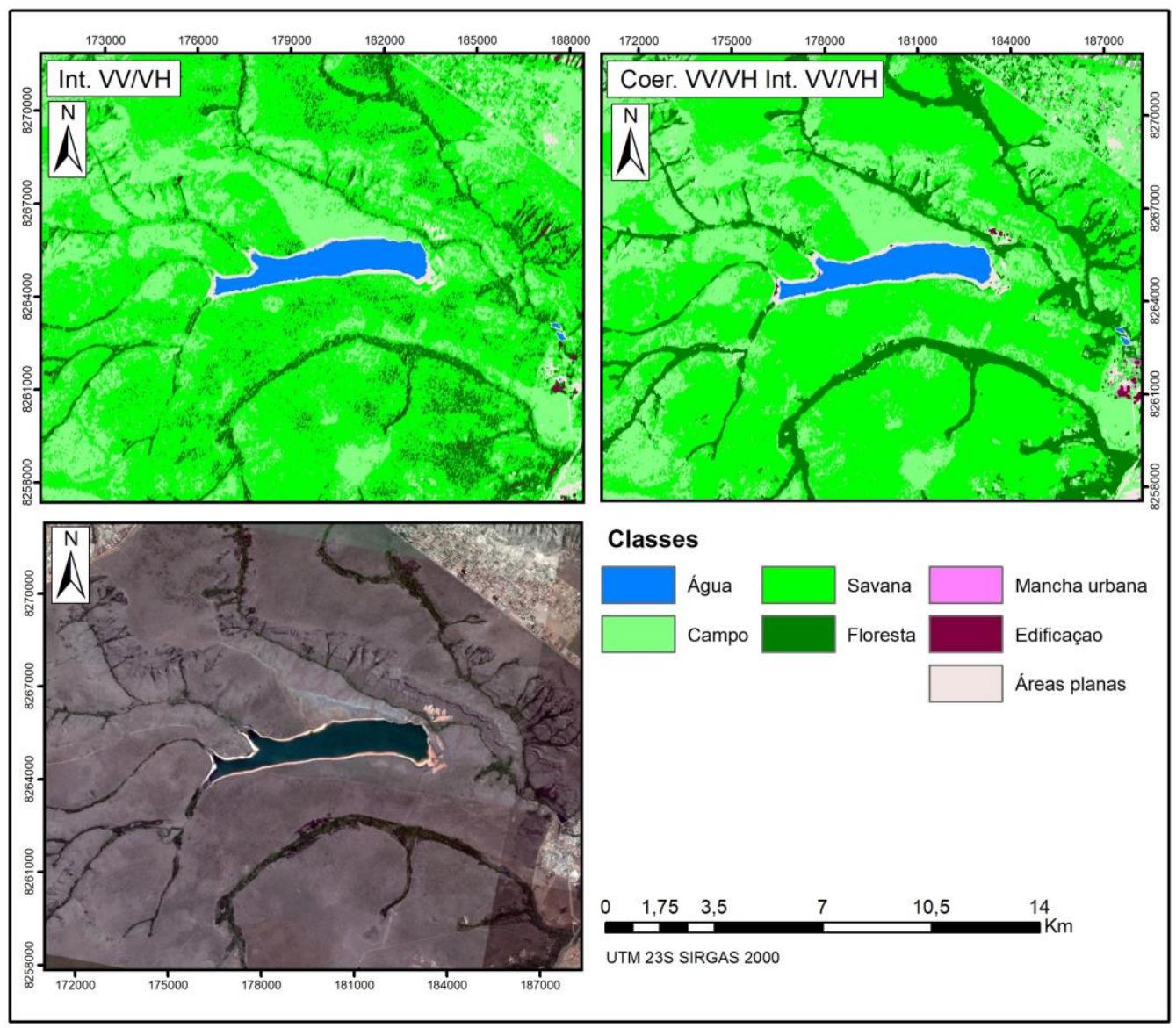

Org:: dos Autores, 2020.

Por sua vez, os resultados apresentados na Figura $8 \mathrm{f}$ indicam um incremento da classe "edificação" de 0,55 a 0,68 na métrica F1. Conforme a Figura 9f, percebe-se que os aumentos dos valores de precision $(0,50$ a 0,71$)$ e de recall $(0,48$ a 0,66$)$ estão associados ao mapeamento de uma classe temática mais genérica na área de estudo, o que não indica, necessariamente, uma melhor definição dos objetos urbanos. A Figura 11 mostra a razão entre os valores de Verdadeiro Positivos (VP) referentes à classe temática "edificação" e as áreas que foram classificadas considerando os resultados (i) utilizando-se apenas as informações de intensidade (VV e VH) e (ii) o agregado de coerências interferométricas e de intensidades em ambas as polarizações. 
Figura 11. Razão entre os valores de VP e as áreas classificadas da classe "edificações".

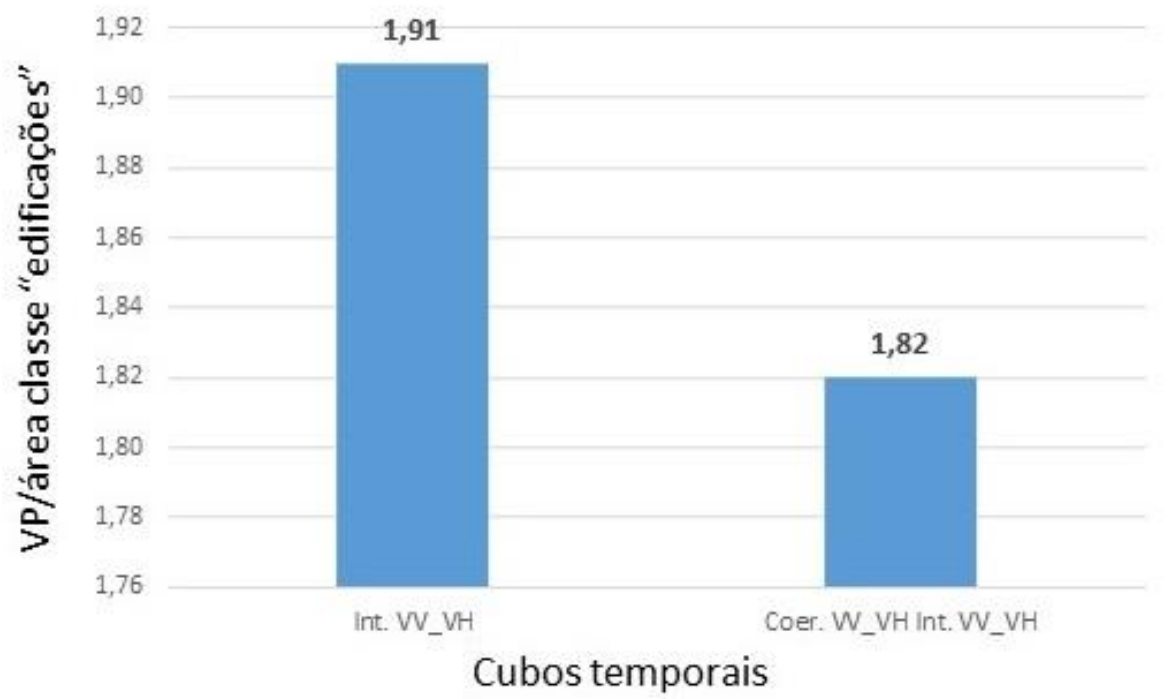

Org.: dos Autores, 2020.

$\mathrm{Na}$ Figura 11, os resultados indicam que a razão para a respectiva classe temática "edificações" é maior na classificação proveniente da série temporal contendo apenas as métricas de intensidade do que considerando toda a dimensionalidade de dados, o que se mostra mais adequada na identificação das áreas com efeito predominantemente double bounce. Também constata-se que a coerência interferométrica, apesar de apresentar melhores índices de acurácia, tende a potencializar o resultado com o aumento de área, ou generalização da classe no processo de classificação do uso e ocupação da terra.

\section{Compilação do mapa de uso e ocupação da terra}

A composição do mapa de uso e ocupação da terra foi resultante da agregação espacial das classes temáticas provenientes da classificação que apresentou o melhor desempenho global nos índices Kappa e F1. A única exceção foi relativa à classe "edificações", que foi proveniente da classificação considerando apenas as intensidades em VV e VH. A Figura 12 apresenta duas regiões da área de estudo visando exemplificar o refinamento realizado pelo processo de agregação espacial dos melhores desempenhos temáticos identificados.

Percebe-se que o método de composição proposto corrigiu a definição dos objetos urbanos no mapa de uso e ocupação da terra estabelecido, garantindo o registro mais fidedigno das regiões caracterizadas por efeito double bounce relacionados com os objetos urbanos. A Figura 13 apresentada o mapa resultante de uso e ocupação da terra estabelecido pela metodologia proposta no presente estudo. 
Figura 12. Exemplos de refinamento da temática urbana - regiões do (a) Guará e do (b) Plano Piloto

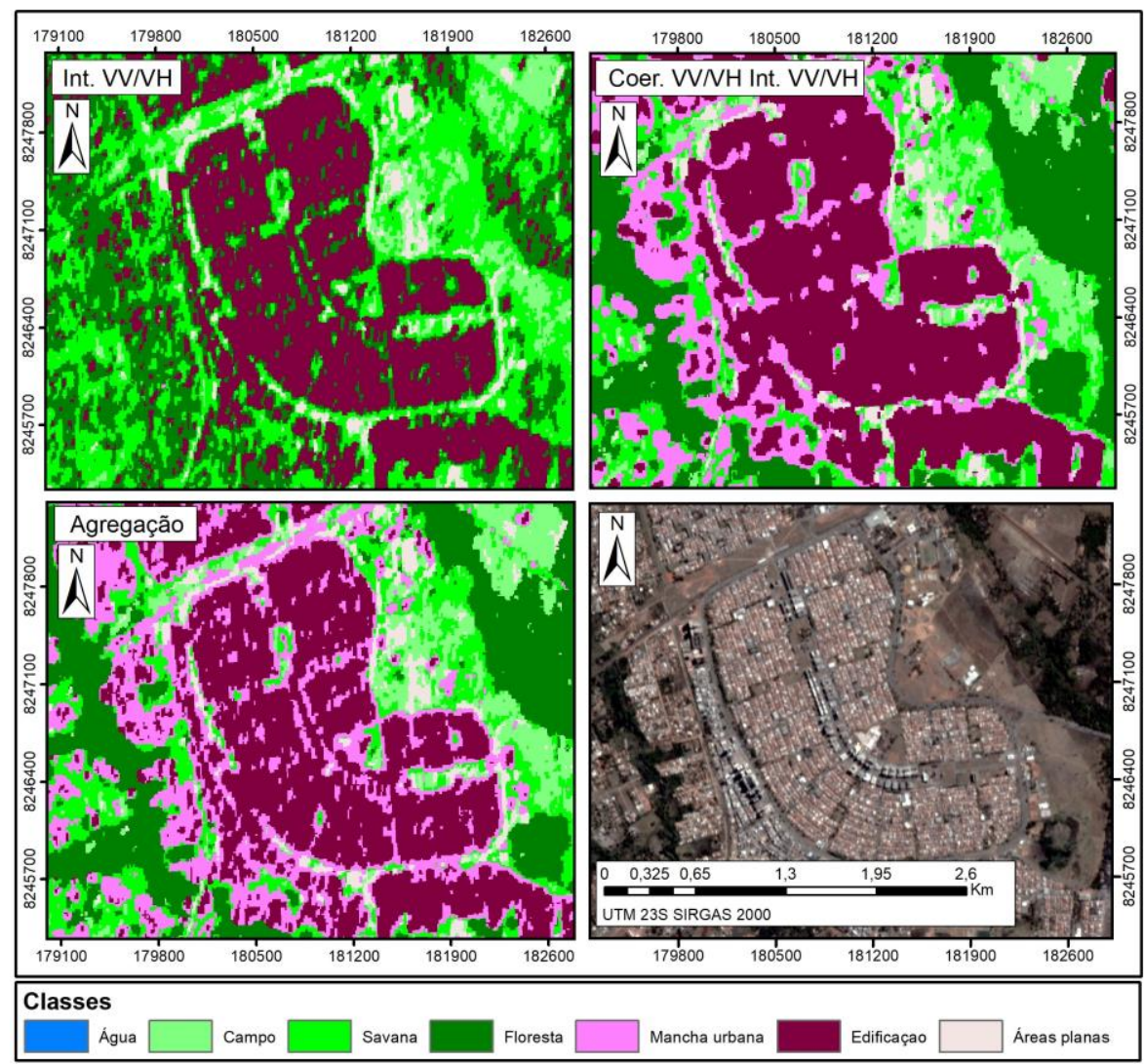

(a)

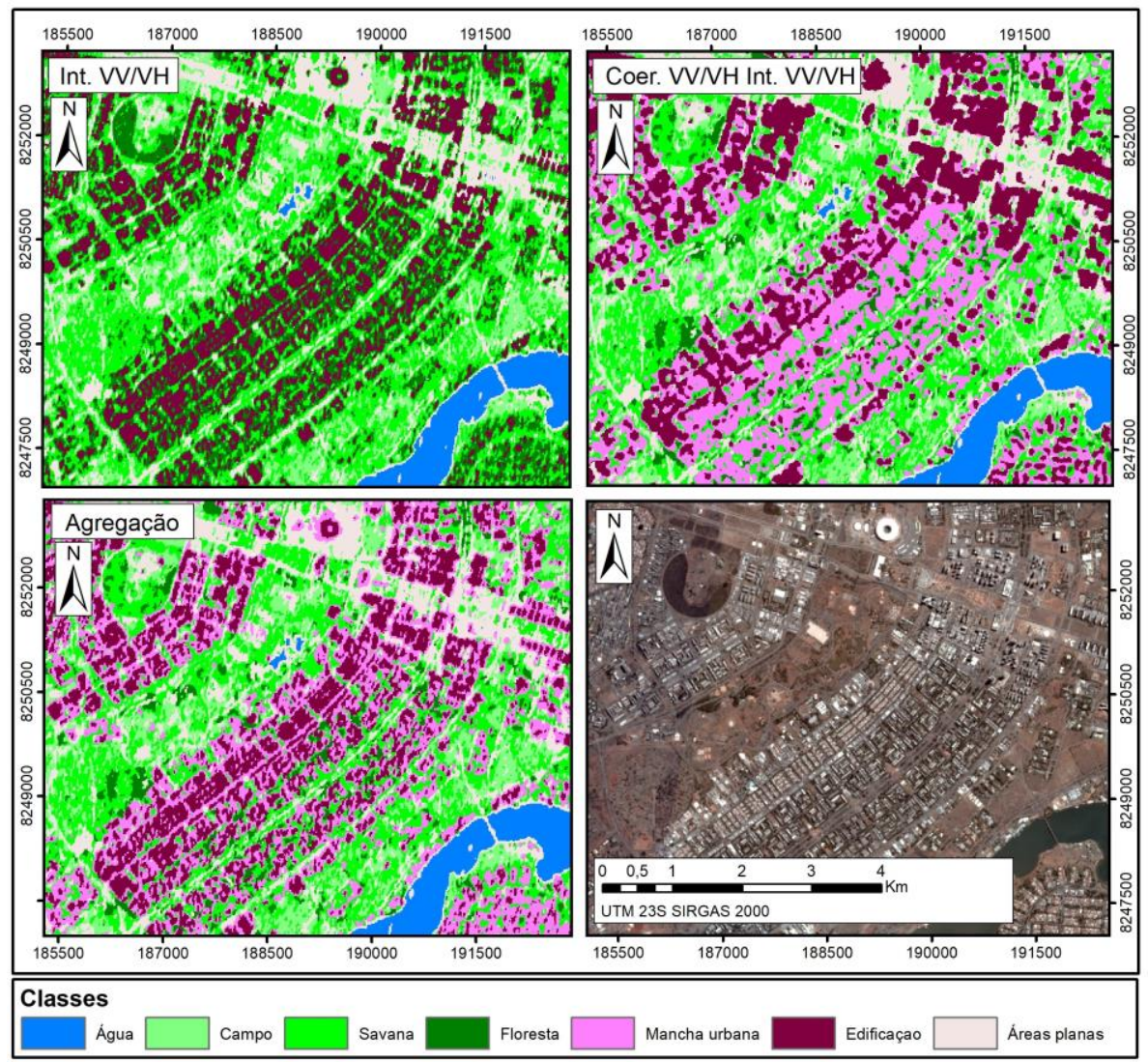

(b)

Org.: dos Autores, 2020 
Figura 13. Mapa compilado de uso e ocupação da terra.

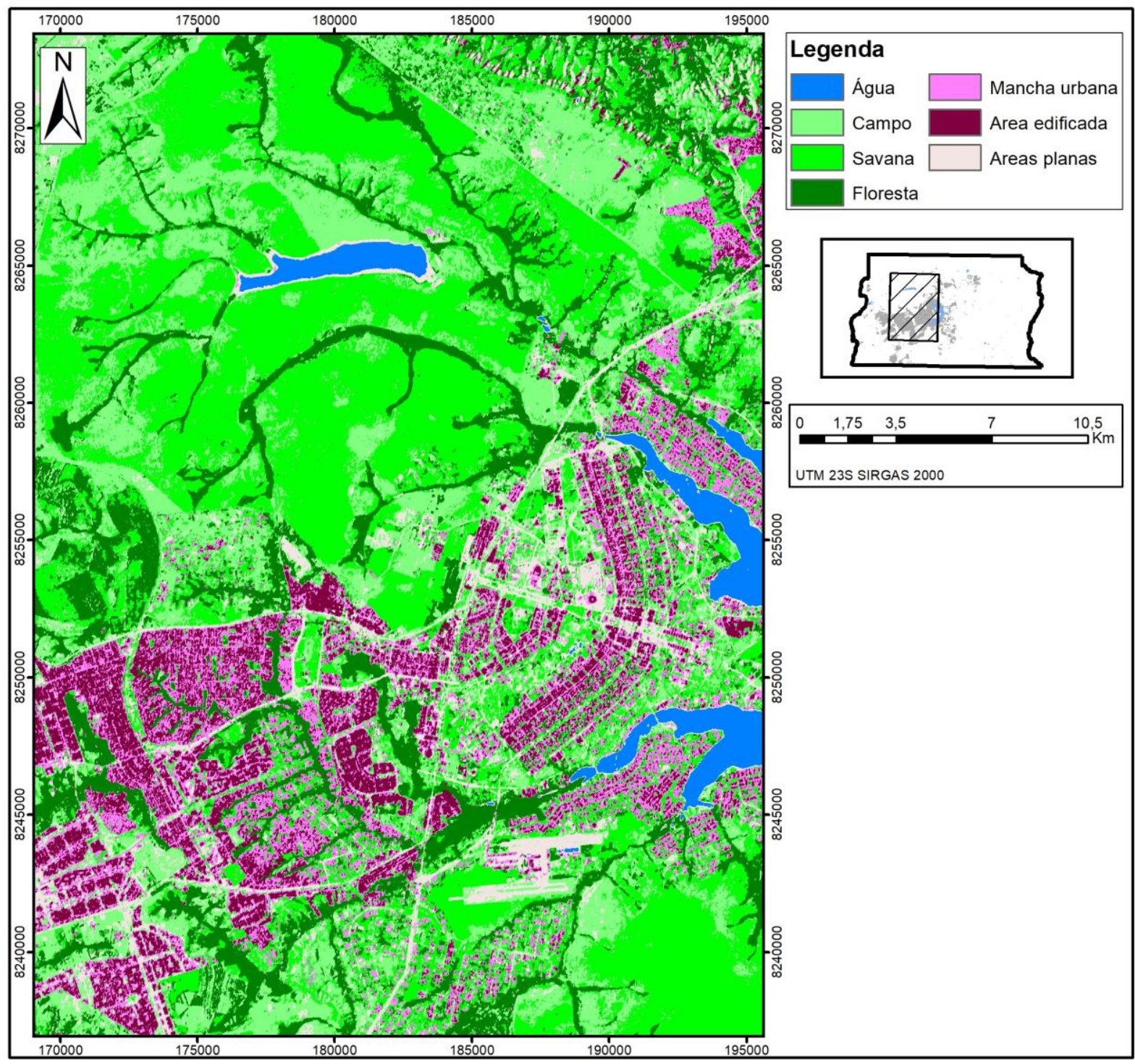

Org.: dos Autores, 2020.

\section{CONSIDERAÇÕES FINAIS}

Esse estudo inova ao apresentar uma abordagem metodológica para o mapeamento do uso e cobertura da terra utilizando as métricas da intensidade e da coerência interferométrica provenientes de uma série temporal de imagens Sentinel 1 SLC. As métricas de acurácia consideradas indicam um aprimoramento de 0,50 a 0,75 (Kappa) e 0,54 a 0,79 (F1) nas classificações com a inserção da coerência interferométrica em série temporal, apresentando um melhor balanceamento entre as métricas precision e recall.

Essa melhora é mais presente nas classes temáticas relacionadas à cobertura vegetal, principalmente naquela que possui maior densidade vegetacional (Floresta), cujos índices variaram de 0,47 a $0,93(\mathrm{~F} 1)$, fato que indica a importância dessa informação SAR como complemento na melhora de desempenho da classificação.

Por sua vez, os resultados indicam que tal inserção prejudica a capacidade dos classificadores em identificar especificamente objetos urbanos, porque a coerência produz a suavização dos valores das métricas SAR, o que não é favorável a classificação de alvos geométricos porque estes possuem alta frequência. Dessa forma, essa classe foi determinada utilizando apenas as métricas de intensidade, em ambas as polarizações. Sendo assim, a compilação das metodologias para a elaboração do mapeamento do uso e cobertura da terra identifica com maior precisão tanto às classes naturais como a definição geométrica dos objetos urbanos. 
Demais estudos podem ser desenvolvidos no sentido de aprimorar o processo de classificação utilizando classificadores orientados a objetos, fusão de sensores óticos e de radar, utilização de aprendizado de máquina profunda (Deep Learning), a partir de cubos temporais que consideram a variação temporal.

\section{REFERÊNCIAS}

AUBLANC, J.; MOREAU, T.; THIBAUT, P.; BOY, F.; RÉMY, F.; PICOT, N. Evaluation of SAR altimetry over the Antarctic ice sheet from CryoSat-2 acquisitions. Advances in Space Research, v. 62, n. 6, p. 1307-1323, 2018 . https://doi.org/10.1016/j.asr.2018.06.043

BELGIU, M.; DRAGUT, L. Random forest in remote sensing: A review of applications and future directions. ISPRS Journal of Photogrammetry and Remote Sensing, v. 114, p. 24-31, 2016. https://doi.org/10.1016/j.isprsjprs.2016.01.011

BITENCOURT, M. D. Classificação espectro-temporal da cultura de grãos em imagens Sentinel (SAR) utilizando Machine Learning em Luís Eduardo Magalhães, Brasil. (Dissertação de Mestrado), Curso de Pós-graduação em Geografia, Universidade de Brasília, 2020, 39f. https://repositorio.unb.br/

BRASIL. Governo de Distrito Federal - GDF. Zoneamento Ecológico-Econômico do Distrito Federal (ZEE/DF). Brasília, 2018. http://www.zee.df.gov.br/

BRASIL. Instituto Brasileiro de Geografia e Estatística - IBGE. Resolução n01/2015 que trata da transformação entre os referenciais geodésicos no Brasil. Rio de Janeiro, 2015.

ftp://geoftp.ibge.gov.br/metodos_e_outros_documento S_de_referencia/normas/rpr_01_2015_sirgas2000.pdf

BREIMAN, L. Random Forests. Machine Learning, v. 45, n. 1, p. 5-32, 2001. https://doi.org/10.1023/A:1010933404324

CAO, R.; TU, W.; YANG, C.; LI, Q.; LIU, J.; ZHU, J.; ZHANG, Q.; LI, Q.; QIU, G. Deep learning-based remote and social sensing data fusion for urban region function recognition. ISPRS Journal of Photogrammetry and Remote Sensing, v. 163, p. 8297, 2020.

https://doi.org/10.1016/j.isprsjprs.2020.02.014

CHATURVEDI, S. K.; BANERJEE, S.; LELE, S. An assessment of oil spill detection using Sentinel 1 SAR-C images. Journal of Ocean Engineering and Science, in press, 2019. https://doi.org/10.1016/j.joes.2019.09.004

CIAMPALINI, A.; SOLARI, L.; GIANNECCHINI, R.; GALANTI, Y.; MORETTI, S. Evaluation of subsidence induced by long-lasting building load using InSAR technique and geotechnical data: The case study of a Freight Terminal (Tuscany, Italy). International Journal of Applied Earth Observation $\begin{array}{llll}\text { and Geoinformation, } & \text { v. } & 82, \quad 2019\end{array}$ https://doi.org/10.1016/j.jag.2019.101925

COSTA, D. H. Uso de séries temporais Sentinel 1 na identificação de culturas agrícolas utilizando modelos de Machine Learning. (Dissertação de Mestrado), Curso de Pós-graduação em Geografia,
Universidade de Brasília, 2020, 51f. https://repositorio.unb.br/

DE ZAN, F.; GUARNIERI, A. M. TOPSAR: Terrain Observation by Progressive Scans. IEEE Transactions on Geoscience and Remote Sensing, v. 44, n. 9, p. 2352-2360, 2006. https://doi.org/10.1109/TGRS.2006.873853

DINIZ, J. M. F. S; GAMA, F. F. Utilização da coerência interferométrica SAR para mapeamento do uso e cobertura da terra na região da Amazônia. In: Simpósio Brasileiro de Sensoriamento Remoto, 2019, Santos/SP. Anais... São José dos Campos: INPE, 2019. Disponível em: https://proceedings.science/sbsr

2019/papers/utilizacao-da-coerencia interferometrica-sar-para-mapeamento-do-uso-ecobertura-da-terra-na-regiao-da-amazonia

FERREIRA, G. H. S. Identificação de áreas inundáveis na porção sul de Roraima com auxílio de imagens de radar. (Dissertação de Mestrado), Curso de Pósgraduação em Geografia, Universidade de Brasília, 2018 ,

$75 f$. https://repositorio.unb.br/handle/10482/33928

GRIFFITHS, P.; HOSTERT, P.; GRUEBNER, O.; LINDEN, V. Mapping megacity growth with multisensor data. Remote Sensing of Environment, v. 114, n. 2, p. 426-439, 2010. https://doi.org/10.1016/j.rse.2009.09.012

HU, L.; DAI, K.; XING, C.; LI, Z.; TOMÁS, R.; CLARK, B.; SHI, X.; CHEN, M.; ZHANG, R.; QIU, Q.; LU, Y. Land subsidence in Beijing and its relationship with geological faults revealed by Sentinel 1 InSAR observations. International Journal of Applied Earth Observation and Geoinformation, v. 82, 2019. https://doi.org/10.1016/j.jag.2019.05.019

KHALIL, R. Z.; HAQUE, S. Insar coherence-based land cover classification of Okara, Pakistan. The Egyptian Journal of Remote Sensing and Space Sciences. v. 21, p. S23-S28, 2018.

https://doi.org/10.1016/j.ejrs.2017.08.005

LAWRENCE, R. L.; MORAN, C. J. The AmericaView Classification Methods Accuracy Project: A Rigorous Approach for Model Selection. Remote Sensing of Environment, v. 170, p. 115-120, 2015. https://doi.org/10.1016/j.rse.2015.09.008

LI, M.; ZHANG, L.; DING, C.; LI, W.; LUO, H.; LIAO, M.; XU, Q. Retrieval of historical surface displacements of the Baige landslide from timeseries SAR observations for retrospective analysis of the collapse event. Remote Sensing of Environment, v. $240,2020$.

https://doi.org/10.1016/j.rse.2020.111695

MARQUES, J. B.; CARVALHO JÚNIOR, O. A.; CAMPAGNOLI, F.; MESQUITA JÚNIOR, H. N.; GOMES, R. A. T.; GUIMARÃES, R. F. Classificação da cobertura da terra na região da ilha do Bananal usando imagens multitemporais PALSAR-2/ALOS2. Revista Franco-Brasileira de Geografia (Confins), v. 39, 2019. https://doi.org/10.4000/confins.17506

MAXWELL, A. E.; WARNER, T. A.; FANG, F. Implementation of machine-learning classification in remote sensing: an applied review. International Journal of Remote Sensing, v. 39, n. 9, p. 2784-2817, 2018. https://doi.org/10.1080/01431161.2018.1433343

MIGUEL, B. H.; SANO, E. E. Classificação do uso e cobertura da terra do Distrito Federal, Brasil a partir de dado de coerência interferométrica. 
Revista Brasileira de Geografia Física, v. 12, n. 2, p. 427-442,

2019.

https://doi.org/10.26848/rbgf.v12.2.p427-442

MOHAMMADIMANESH, F.; SALEHI, B.; MAHDIANPARI, M.; BRISCO, B.; MOTAGH, M. Multi-temporal, multi-frequency, and multipolarization coherence and SAR backscatter analysis of wetlands. ISPRS Journal of Photogrammetry and Remote Sensing, v. 142, p. 7893, 2018.

https://doi.org/10.1016/j.isprsjprs.2018.05.009

OLESK, A.; PRAKS, J.; ANTROPOV, O.; ZALITE, K.; ARRUMAE, T.; VOORMANSIK, K. Interferometric SAR Coherence Models for Characterization of Hemiboreal Forests Using TanDEM-X Data. Remote $\begin{array}{llllll}\text { Sensing, } & \text { v. } & 8, & \text { n. } & 9, & 2016 .\end{array}$ https://doi.org/10.3390/rs8090700

OLIVEIRA, P. D. S. Uso de aprendizagem de máquina e redes neurais convolucionais profundas para a classificação de áreas queimadas em imagens de alta resolução espacial. (Dissertação de Mestrado), Curso de Pós-graduação em Geografia, Universidade de Brasília, 2019, 34p. https://repositorio.unb.br

OZIGIS, M. S.; KADUK, J. D.; JARVIS, C. H.; BISPO, P. C.; BALZTER, H. Detection of oil pollution impacts on vegetation using multifrequency SAR, multispectral images with fuzzy forest and random forest methods. Environmental Pollution, v. 256, 2020.

https://doi.org/10.1016/j.envpol.2019.113360

PICHIERRI, M.; HAJNSEK, I.; ZWIEBACK, S.; RABUS, B. On the potential of Polarimetric SAR Interferometry to characterize the biomass, moisture and structure of agricultural crops at L-, C- and X-Bands. Remote Sensing of Environment, v. 204, p. 596-616, 2018. https://doi.org/10.1016/j.rse.2017.09.039

PRINZIE, A; VAN DEN POEL, D. Random Forests for multiclass classification: Random MultiNomial Logit. Expert systems with Applications: An International Journal, v. 34 , n. 3, p. 1721-1732, 2008. https://doi.org/10.1016/j.eswa.2007.01.029

RAJAH, P.; ODINDI, J.; MUTANGA, O. Feature level image fusion of optical imagery and Synthetic Aperture Radar (SAR) for invasive alien plant species detection and mapping. Remote Sensing Applications: Society and Environment, v. 10, p. 198-208, 2018. https://doi.org/10.1016/j.rsase.2018.04.007

SALGADO, C. B. Emprego de séries temporais na Amazônia: análise de imagens MODIS e RADAR para mapeamento de uso e ocupação do solo no Estado do Acre. (Tese de Doutorado), Curso de Pósgraduação em Geografia, Universidade de Brasília, 2019, $113 \mathrm{f}$. https://repositorio.unb.br/handle/10482/35999

SALGADO, C. B.; CARVALHO JÚNIOR, O. A.; GOMES, R. A. T.; GUIMARÃES, R. F. Análise da interferência de nuvens na classificação de séries temporais MODIS-NDVI na região da Amazônia, município de Capixaba, Acre. Sociedade \& Natureza, v. 31, p. 1-20, 2019.

https://doi.org/10.14393/SN-v31-2019-47062

SEKERTEKIN, A.; MARANGOZ, A. M.; ADBIKAN, S. ALOS-2 and Sentinel-1 SAR data sensitivity analysis to surface soil moisture over bare and vegetated agricultural fields. Computers and Electronics in Agriculture, v. 171, 2020. https://doi.org/10.1016/j.compag.2020.105303

SICA, F.; PULELLA, A.; NANNINI, M.; PINHEIRO, M.; RIZZOLI, P. Repeat-pass SAR interferometry for land cover classification: A methodology using Sentinel-1 Short-Time-Series. Remote Sensing of Environment, $\quad$ v. 2019. https://doi.org/10.1016/j.rse.2019.111277

WEGMULLER, U.; SANTORO, M.; WERNER, C.; CARTUS, O. On the estimation and interpretation of Sentinel 1 TOPS InSAR coherence. In: Proc. of FRINGE 2015 Workshop, Frascati, Italia, 23-27 março, ESA, 2015. http://proceedings.esa.int/files/89.pdf

WERNER, A.; STORIE, C. D.; STORIE, J. Evaluating SAR-Optical Image Fusions for Urban LULC Classification in Vancouver Canada. Canadian Journal of Remote Sensing, v. 40, n. 4, p. 278-290, 2014. https://doi.org/10.1080/07038992.2014.976700

WHITCOMB, J.; MOGHADDAM, M.; MCDONALD, K.; KELLNDORFER, J.; PODEST, E. Mapping vegetated wetlands of Alaska using L-band radar satellite imagery. Canadian Journal of Remote Sensing, v. 35, n. 1, p. 54-72, 2009. https://doi.org/10.5589/m08-080

ZHANG, H.; LI, J.; WANG, T.; LIN, H.; ZHENG, Z.; LI, Y.; LU, Y. A manifold learning approach to urban land cover classification with optical and radar data. Landscape and Urban Planning, v. 172, p. 11-24, 2018. https://doi.org/10.1016/j.landurbplan.2017.12.009

ZHU, Z.; ZHOU, Y.; SETO, K. C.; STOKES, E. C.; DENG, C.; PICKETT, S. T. A.; TAUBENBOCK, H. Understanding an urbanizing planet: Strategic directions for remote sensing. Remote Sensing of Environment, v. 228, p. 164-182, 2019. https://doi.org/10.1016/j.rse.2019.04.020

\section{Contribuições dos Autores}

Felipe Lima Ramos Barbosa e Renato Fontes Guimarães conceberam o estudo, analisaram os dados e redigiram o texto. Felipe Lima Ramos Barbosa coletou os dados e processou as informações e Renato Fontes Guimarães orientou o andamento do trabalho. Osmar Abílio de Carvalho Júnior e Roberto Arnaldo Trancoso Gomes avaliaram os resultados e garantiram a consistência dos dados, como também corrigiram o texto produzido.

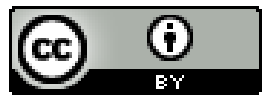

Este é um artigo de acesso aberto distribuído nos termos da Licença de Atribuição Creative Commons, que permite o uso irrestrito, distribuição e reprodução em qualquer meio, desde que o trabalho original seja devidamente citado. 